\title{
A SPLITTING THEOREM FOR THE N-R.E. DEGREES
}

\author{
S. BARRY COOPER
}

(Communicated by Andreas R. Blass)

\begin{abstract}
We prove a splitting theorem for the n-r.e. degrees, of which the Sacks Splitting Theorem [9] for the r.e. (=1-r.e.) degrees is a special case. For background terminology and notation see [4] and [11].
\end{abstract}

Interest in the n-r.e. (and more particularly, d-r.e. $=2$-r.e.) degrees stems from their affinity even for large $n$ with the r.e. degrees, and a number of recent papers (see for example $[1,2,4,6]$ ) have sought to clarify to what extent there are similarities and differences at the individual levels of the n-r.e. degree hierarchy. Expectations that the few global properties of the r.e. degrees (in particular density) might carry over to the n-r.e. degrees, $n>1$, have been only partially fulfilled. Lachlan observed that n-r.e. degrees are not minimal in the n-r.e. degrees, essentially because they are n-REA (see [7]), but density fails, even under $\mathbf{0}^{\prime}$ (see [3]). On the other hand, some of the pathological properties of the r.e. degrees disappear in the wider context, such as in Downey's diamond theorem [5] for the d-r.e. degrees and Arslanov's use [1] (and more generally in [4]), of d-r.e. degrees for cupping. Therefore the theorem below is welcome in the sense that we now have a global splitting property at every level of the n-r.e. hierarchy of degrees. (We note that the splitting of the n-r.e. degrees in the $\Delta_{2}$ degrees is an immediate consequence of the n-REA property and the relativised Sacks Splitting Theorem.) Further information relating to the structure of the n-r.e. degrees may be found in [8].

Theorem. Let $\mathbf{d}$ be $n$-r.e. Then there exist $n$-r.e. degrees $\mathbf{a}, \mathbf{b}$ such that $\mathbf{a} \mid \mathbf{b}$ and $\mathbf{a} \cup \mathbf{b}=\mathbf{d}$.

In order to prove the theorem, we give a construction for the case d properly d-r.e. and then indicate how our construction can be adapted to give the inductive step in the proof of the theorem for all $n \geq 1$. The case $n=1$ is just the Sacks Splitting Theorem, of course.

Let $D \in \mathrm{d}$ be d-r.e. We construct d-r.e. sets $A, B$, and partial recursive (p.r.) functionals $\Gamma, \Lambda$, and $\Omega$, satisfying the overall requirements

$$
A=\Gamma^{D}, \quad B=\Lambda^{D}, \quad D=\Omega^{A, B}
$$

Received by the editors June 28, 1989 and, in revised form, September 12, 1990.

1991 Mathematics Subject Classification. Primary 03D25.

Research supported by a Consiglio Nazionale delle Riscerche Visiting Professorship and by the Mathematics Department of the Università di Siena, April-June 1989. 
and the prioritised list of requirements

$$
\begin{aligned}
R_{2 i}: D & =\Phi_{i}^{A} \Rightarrow\left(\exists I_{2 i} \text { r.e. }\right) \quad I_{2 i} \equiv_{T} D, \\
R_{2 i+1}: D & =\Phi_{i}^{B} \Rightarrow\left(\exists I_{2 i+1} \text { r.e. }\right) \quad I_{2 i+1} \equiv_{T} D,
\end{aligned}
$$

$i \geq 0$, where $\left\{\Phi_{i}\right\}_{i \geq 0}$ is a standard listing of the p.r. functionals. The overall requirements will ensure that $A, B \leq_{T} D$ and $D \leq_{T} A, B$, so $D \equiv_{T} A \oplus B$. We use the convention that if $\Theta$, say, is a p.r. functional then small $\theta$ denotes its standard use function.

Our strategy is initially the Sacks Splitting strategy with a few modifications. The main new feature is in the way the $R$-requirements are set up and satisfied.

The splitting strategy is almost as one would expect. As numbers are enumerated into $D$ traces for these numbers permit $x \in D$ by being enumerated into $A$ or $B$, but not both (a trace for $x$ being a number whose presence in $A$ or $B$ indicates that $x \in D$ ). We cannot just use the number itself as the trace though, as we may need to change our mind about whether it is to be in $A$ or $B$ more than once, and $A, B$ must be d-r.e. If such a number is subsequently extracted from $D$, its current trace must also be extracted from $A \cup B$. As in the Sacks construction, we do have freedom of choice when $x \searrow D$ (that is, $x$ enters $D)$ as to whether to change $A \uparrow \omega(x)$ or $B \uparrow \omega(x)$ in enumerating the current trace for $x$ into $A \cup B$, and we exercise this choice in the interests of avoiding injuring requirements of relatively higher priority.

There is no choice, however, when it comes to extracting the trace of $x$ from $A \cup B$. Injury to the $R_{2 i}$ or $R_{2 i+1}$ requirements is usually immediate and unavoidable. But the discretion exercised in enumerating into $A$ and $B$ together with the usual Sacks restraints over $l\left(D, \Phi_{i}^{A}\right), l\left(D, \Phi_{i}^{B}\right)$ (that is, the standard lengths of agreement at the appropriate stage of the construction) provide us with (in Soare's terminology [11, p. 112]) an injury set for a particular requirement that is r.e. instead of d-r.e. This results in $D \neq \Phi_{i}^{A}\left(\right.$ and $D \neq \Phi_{i}^{B}$ ) since otherwise we would have $D \equiv_{T}$ a r.e. $I_{2 i}$ (or $I_{2 i+1}$ ) contradicting the properly d-r.e.-ness of $\mathbf{d}$.

The main source of complication in this simple outline is that there is a new infinite outcome in which $D \neq \Phi_{i}^{A}$, say, through $\Phi_{i}^{A}(x) \uparrow$ (that is, $\Phi_{i}^{A}(x)$ is undefined) for some $x$, where $l\left(D, \phi_{i}^{A}\right)$ is unbounded, perhaps. This is because, in contrast to the Sacks splitting theorem where the prioritisation gives us a finite (that is, 0-r.e.) injury set for a 1-r.e. splitting, here the reduction is of a 2-r.e. splitting to a 1-r.e. (that is, r.e.) injury set.

The reason why we can still operate our strategy is that the $A$-use of $l\left(D, \Phi_{i}^{A}\right)$ must have bounded lim inf. But this fact will only emerge over a period, and since there may be a number of such lim inf's to consider in deciding which of $A$ or $B$ is to permit $x \searrow D$, there will be a number of false permissions decided upon and then extracted. Eventually of course, in the usual way, we identify the true path relative to $x \searrow D$ and make the correct choice of $A \uparrow \omega(x)$ or $B \uparrow \omega(x)$ change. So the Sacks strategy is still intact, but now buried in a tree of strategies.

In the construction below, we assume that for each $x$ we have available a fixed, previously chosen, finite set of $A$ - and $B$-traces for $x$. We will only have regard for the first $x$ requirements in deciding whether to record $x \searrow D$ in $A$ or $B$. This will still mean that $R_{x}$ will be given due regard in deciding whether to use $A$ - or $B$-traces for $y>x$, which is enough. But it will also 
mean that if we take care in describing those initial segments of agreement $l\left(D, \Phi_{i}^{A}\right)$ or $l\left(D, \Phi_{i}^{B}\right)$, which we protest at later stages of the construction (see the definition of fixations for $A$ and $B$ below), then we can ensure that we need at most $(x+1) ! A$ and $B$ traces available for recording $x \backslash D$ in $A \cup B$. This determines the size of the finite sets of $A$ - and $B$-traces for $x, x \geq 0$, allowing us to keep all such traces $\leq \omega(x)$ at all stages of the construction. (For convenience, we also choose each trace for $x$ to be $\geq x$.)

Of course, each time we change our mind about the current trace $y$ for $x$ (whether to choose an $A$ - or $B$-trace usually) this will usually mean we need a $D\lceil\gamma(y)$ or $D\lceil\lambda(y)$ change to permit this via $\Gamma$ or $\Lambda$. We will see in the construction and proof below that we will only want to switch trace for $x$ when certain $D$-changes change the relationship of $x$ to one or more of the requirements, and the definitions of $\gamma(y), \lambda(y)$ will be tailored to take advantage of these changes.

It will be useful to relate our construction to a tree of outcomes. We first define the following auxiliary functions (using the convention that $X \mid z=$ $X(0) X(1) \cdots X(z-1)$ for each set $X \subseteq \omega$, each $z \geq 0)$

$$
\begin{aligned}
l^{s}\left(D, \Phi_{i}^{X}\right) & =\mu z\left[\Phi_{i}^{X}(z) \neq D(z) \text { at stage } s\right], \\
m\left(D, \Phi_{i}^{X}\right) & =\max \left\{l^{t}\left(D, \Phi_{i}^{X}\right) \mid t \leq s\right\}, \\
u^{s}\left(\Phi_{i}^{X}, x\right) & =\mu w\left[\Phi_{i}^{X \nmid w+1}\lceil x \downarrow \text { at stage } s],\right. \\
u^{s}\left(\Phi_{i}^{X}\right) & =u^{s}\left(\Phi_{i}^{X}, l^{s}\left(D, \Phi_{i}^{X}\right)\right) .
\end{aligned}
$$

We say $x$ is a $t$-fix if and only if $x \in D^{t}-D^{t-1}$.

We say $y$ is a $t$-fix trace (at stage $s+1$ ) if and only if $y$ is the current trace of some $t$-fix.

At stage $s+1$ of the construction, as well as defining $A$ and $B$, we will define finite sets, called $t$-fixations for $A$ and $B$, written $\left\langle t A^{s+1}\right\rangle$ and $\left\langle t B^{s+1}\right\rangle$ (or just $\langle t A\rangle$ and $\langle t B\rangle$ when we are just referring to their current values), where $\left\langle t A^{s+1}\right\rangle$ and $\left\langle t B^{s+1}\right\rangle$ will consist of $A^{s+1}$ and $B^{s+1}$, respectively, minus those $t^{\prime}$-fix traces, $t^{\prime}>t$ that have been allocated to $A$ or $B$, respectively, at stage $s+1$. The definition of $t$-fixations is motivated by the need to update our actions in assigning $t$-fix traces to $A$ or $B$ in the light of the changing state of the restraints needed to satisfy the $R$-requirements. This updating (see the definition of trace location exercise in stage $s+1$ of the construction below) must be inductive, as how we deal with a $t$-fix trace may change our assessment of which restraints to retain at stage $s=1$, and hence how to assign at stage $s+1$ a $t^{\prime}$-fix trace for some $t^{\prime}>t$. So the finite sets $\left\langle t A^{s+1}\right\rangle$ and $\left\langle t B^{s+1}\right\rangle$ are used to record the steps in this inductive definition of $A^{s+1}$ and $B^{s+1}$.

We also need some terminology to describe whether a use $u^{t}\left(\Phi_{i}^{A}, z\right)$ observed at a stage $t$ is still a candidate for being restrained at a later stage $s+1$. We say $u^{t}\left(\Phi_{i}^{A}, z\right)$ is valid (at stage $\left.s+1\right)$ if and only if $A^{t} \mid u^{t}\left(\Phi_{i}^{A}, z\right) \subset\left\langle t A^{s+1}\right\rangle$ (set inclusion here), and define

$$
\begin{aligned}
& \mathscr{U}^{t, s+1}\left(\Phi_{i}^{A}\right)= \max \left\{u^{t}\left(\Phi_{i}^{A}, z\right) \mid z \leq l^{t}\left(D, \Phi_{i}^{A}\right)\right. \\
&\left.\& u^{t}\left(\Phi_{i}^{A}, z\right) \text { is valid at stage } s+1\right\}, \\
& \mathscr{U}^{s+1}\left(\Phi_{i}^{A}\right)=\max \left\{\mathscr{U}^{t, s+1}\left(\Phi_{i}^{A}\right) \mid t \leq s+1\right\} .
\end{aligned}
$$

There are similar definitions with $B$ in place of $A$. 
Then for each $R_{2 i}$ and each $n \geq 0$, “ $n$ " is the outcome

and for each $R_{2 i+1}$ it is

$$
\text { “ } \lim \inf _{s} \mathscr{U}^{s}\left(\Phi_{i}^{A}\right)=n, "
$$

$$
" \lim \inf _{s} \mathscr{U}^{s}\left(\Phi_{i}^{B}\right)=n . "
$$

The branching at a general vertex of the tree of outcomes looks like

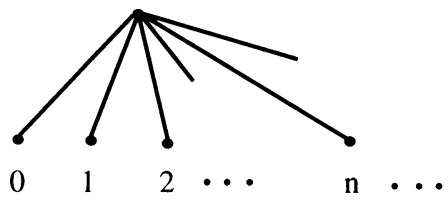

We will use standard terminology (cf. [11]) in regard to the leftmost path and the true path (the leftmost path consisting of leftmost vertices visited infinitely often during the construction, the true path of vertices describing the actual outcome of the construction).

We assume below that for each $s \geq 0, D^{s+1}$ and $D^{s}$ differ on exactly one number.

\section{THE CONSTRUCTION}

Stage 0. Define $A^{0}=B^{0}=\varnothing$.

Stage $s+1$. Case I. $\exists x \in D^{s+1}-D^{s}$.

We set in motion a trace location exercise for this $x$ and say that $x$ has entry stage $s+1$. We suspend the trace location exercise for each $x^{\prime} \in D^{s+1}$ for which $x^{\prime}>x$. (This means that at subsequent stages $t+1$ we allow the trace location exercise for $x$ to decide, in the interest of the $R$-requirements, the optimal placement of all current traces for such numbers $x^{\prime}$, so long as $x \in D^{t+1}$ and the trace location exercise for $x$ is not itself suspended at stage $t+1$. This procedure is necessary to avoid such numbers $x^{\prime}$ leading to the number of trace changes for $x$ becoming unbounded.)

We say a finite string of numbers $\sigma$ is valid (intuitively, to the left of the true path) at stage $s+1$ if and only if for each $y<\ln (\sigma)$

$$
\sigma(y) \leq \begin{cases}\mathscr{U}^{s+1}\left(\Phi_{i}^{A}\right) & \text { if } y=2 i, \text { some } i \geq 0, \\ \mathscr{U}^{s+1}\left(\Phi_{i}^{B}\right) & \text { if } y=2 i+1, \text { some } i \geq 0 .\end{cases}
$$

We write $\sigma_{x}^{s+1}$ for the largest string of length $x$ that is valid at stage $s+1$ (where we order the strings lexicographically upwards).

Intuitively, $\sigma_{x}^{s+1}$ denotes a current guess at the true path. Each true outcome for requirements $R_{y}, y<\ln \left(\sigma_{x}^{s+1}\right)$, appears infinitely often as $\sigma_{x}^{s+1}(y)$, but because these appearances for distinct requirements are not necessarily in phase we actually need a less instantaneous assessment of the true path at stage $s+1$.

We say $\rho$ is $x^{\prime}$-grounded at stage $s+1$ if and only if $\ln (\rho)=x^{\prime}, x^{\prime}$ has entry stage $e+1 \leq s+1$, and for each $y<\ln (\rho)$ we have

$$
\rho(y)= \begin{cases}\mathscr{U}^{e, s+1}\left(\Phi_{i}^{A}\right) & \text { if } y=2 i, \\ \mathscr{U}^{e, s+1}\left(\Phi_{i}^{B}\right) & \text { if } y=2 i+1 .\end{cases}
$$

(So if $\rho$ is $x^{\prime}$-grounded then $\rho$ is valid at stage $s+1$.)

We write $\rho_{x^{\prime}}^{s+1}$ for the unique $x^{\prime}$-grounded string at stage $s+1$, each $x^{\prime} \in$ $D^{s+1}$. 
At each stage $t+1 \geq s+1$, if $x \in D^{t^{\prime}+1}$ some $t^{\prime} \leq t$, the trace location at stage $t+1$ consists of finding the least $y$ (if it exists) such that $x \leq \rho_{x}^{t+1}(y)$ and ensuring that for this $y$ we locate the trace for $x$ in $A$ or $B$ (but not both) in such a way that

$y=2 i$ some $i \Rightarrow$ only the current trace for $x$ is in $\left\langle s+1 A^{t+1}\right\rangle \cup\left\langle s+1 B^{t+1}\right\rangle$, and this is in $\left\langle s+1 B^{t+1}\right\rangle-\left\langle s+1 A^{t+1}\right\rangle$,

and

$$
y=2 i+1 \quad \text { some } i \Rightarrow \text { the same with } A \text { and } B \text { interchanged. }
$$

(If $y$ does not exist, the trace location exercise for $x$ at stage $t+1$ just consists of locating the $x$-trace in $A$, that is, we put the current $x$-trace in $\left\langle s+1 A^{t+1}\right\rangle$.)

Intuitively again, this is where we apply a local (to stage $s+1$ ) version of the Sacks splitting strategy. That is, we check the highest priority requirement likely to be injured by the enumeration of $x$ into $A^{s+1} \cup B^{s+1}$ and protect the restraint associated with this requirement by enumerating the current trace for $x$ (using the fact that $x$ is $\leq$ all its traces) into the appropriate set $A^{s+1}$ or $B^{s+1}$.

If this involves a change of $x$-trace, we extract the existing $x$-trace from $\left\langle s+1 A^{t+1}\right\rangle \cup\left\langle s+1 B^{t+1}\right\rangle$ and choose a new current $x$-trace (which may be chosen to be any as yet unused member of the set of potential traces for $x$ ) and enumerate this into the appropriate set $(\langle s+1 A\rangle$ or $\langle s+1 B\rangle)$.

However, if the trace location exercise for $x$ is suspended, if we extract an existing $x$-trace we do not choose a new current $x$-trace via the trace location exercise for $x$, but choose a new $x$-trace in $A$ or $B$ according as there is an $x^{\prime}$-trace in $A$ or $B$ where $x^{\prime}$ is the unique number $\in D^{t+1}$ through which the trace location for $x$ was suspended, but whose own trace location is not suspended. We continue the situation where the $x$-trace is in the same set ( $A$ or $B)$ as the described $x^{\prime}$-trace until all suspensions of the trace location exercise for $x$ have been cancelled.

At stage $s+1$ we carry out the trace location exercise for each $x^{\prime} \in D^{s^{\prime}}$, some $s^{\prime} \leq s$, in ascending order of entry stage. This will ensure that $\rho_{x}^{s+1}$ is available at stage $s+1$ by the time the trace location for $x$ is due to be carried out. Following the trace location exercise at stage $s+1$, we define for each $t \leq s+1$

$$
\begin{aligned}
\left\langle t A^{s+1}\right\rangle= & \text { the set of all those } t^{\prime} \text {-fix traces located in } A \text { during } \\
& \text { the trace location exercise at stage } s+1, \text { for some } t^{\prime} \leq t, \\
A^{s+1}= & \left\langle s+1 A^{s+1}\right\rangle=\bigcup_{t^{\prime} \leq s+1}\left\langle t^{\prime} A^{s+1}\right\rangle .
\end{aligned}
$$

Case II. $\exists x \in D_{s}-D_{s+1}$.

We extract from $A \cup B$ any $x$-trace in $A \cup B$ at stage $s$.

We also cancel any suspensions of trace location exercise for numbers $x^{\prime} \in$ $D^{s+1}$ imposed at the entry stage of $x$ through Case I. We continue at the next stage the trace location of any $x^{\prime}$ that has had all its suspensions cancelled by the end of stage $s+1$.

\section{New AXIOMS FOR $\Omega, \Gamma$, AND $\Lambda$ AT STAGE $s+1$}

The definition of axioms for $\Omega$ is straightforward. As previously described, we ensure that we choose $\omega(x)$ (as the least number) $\geq$ all $x$-traces at each 
stage and make sure that we define $\Omega^{A, B}(x)=D(x)$ at each stage $s+1>x$.

For $\Gamma$ and $\Lambda$, for each $y \geq 0$ we define $\Gamma^{D}(y)=A(y), \Lambda^{D}(y)=B(y)$ for each stage $s+1>y$.

If $y$ is an $x$-trace for use with $A$ (say), we ask that if $x \notin \bigcup_{t^{\prime} \leq s+1} D^{t^{\prime}}$ then $\gamma(y) \geq x$. If $x \in D^{s+1}$, we define $\gamma^{s+1}(y)$ so that

$$
\begin{array}{r}
\gamma^{s+1}(y)=\max \left\{\rho_{x^{\prime}}^{s+1}(z) \mid x^{\prime} \in D^{s+1}, z<\operatorname{lh}\left(\rho_{x^{\prime}}^{s+1}\right),\right. \\
\left.\& x^{\prime} \text { has entry stage } \leq \text { that of } x\right\} .
\end{array}
$$

(The description, including the definition of $\lambda^{s+1}(y)$, is similar for $\dot{y}$ an $x$-trace for use with $B$.)

The above definitions of $\gamma^{s+1}(y)$ and $\lambda^{s+1}(y)$ are motivated by the need to ensure that whenever we need to relocate an $x$-trace (from $A$ to $B$ say), then the existing $x$-trace maximizes each of the numbers whose extraction from $D$ is likely to result in a variation of the trace location exercise for $x$ at stage $s+1$.

This ends the description of stage $s+1$ of the construction.

Then $A, B$ are defined by

$$
A(x)=\operatorname{Lim}_{s} A^{s}(x), \quad B(x)=\operatorname{Lim}_{s} A^{s}(x),
$$

each $x \geq 0$.

We first check that the construction is well defined. This follows from

Lemma 1. Each number $x$ requires at most $(x+1)$ ! traces during the construction.

Proof. We first notice that an $x$-trace change at stage $s+1$ is due to $\rho_{x}^{s+1}(z) \neq$ $\rho_{x}^{s}(z)$ for some $z<x$. Let $z^{*}$ be the least such $z$, and (without loss of generality) assume $z^{*}=2 i$, for some $i \geq 0$.

So (assuming $x$ has entry stage $e+1$ ) we have

$$
\rho_{x}^{s}\left(z^{*}\right)=\mathscr{U}^{e, s}\left(\Phi_{i}^{A}\right)=u^{e}\left(\Phi_{i}^{A}, w\right),
$$

say, where $u^{e}\left(\Phi_{i}^{A}, w\right)$ is valid at stage $s+1$, and either

(i) there is some $u^{e}\left(\Phi_{i}^{A}, w^{\prime}\right)>u^{e}\left(\Phi_{i}^{A}, w\right), w^{\prime} \leq l^{t}\left(D, \Phi_{i}^{A}\right)$, which is valid at stage $s+1$ but not at stage $s$, or

(ii) $u^{e}\left(\Phi_{i}^{A}, w\right)$ is no longer valid at stage $s+1$.

If (i) holds we have

$$
A^{e} \mid u^{e}\left(\Phi_{i}^{A}, w^{\prime}\right) \subset\left\langle e A^{s+1}\right\rangle, \quad \text { but } \not \subset\left\langle e A^{s}\right\rangle .
$$

This means that for some $t$-fix trace $y$ for some (minimal) $x^{\prime}$ with $t \leq e$, either

$$
y \in\left(A^{e} \mid u^{e}\left(\Phi_{i}^{A}, w^{\prime}\right)-\left\langle e A^{s}\right\rangle\right) \cap\left\langle e A^{s+1}\right\rangle
$$

or

$$
y \in\left\langle e A^{s}\right\rangle \uparrow u^{e}\left(\Phi_{i}^{A}, w^{\prime}\right)-\left(A^{e} \cup\left\langle e A^{s+1}\right\rangle\right) .
$$

Then either $y$ is relocated at stage $s+1$ or $x^{\prime} \in D^{s}-D^{s+1}$. In either case, since $x^{\prime} \leq y$, we have $x^{\prime} \leq u^{e}\left(\Phi_{i}^{A}\right)$.

Also, by the construction, in the former case we have $t<e$, so that we must have $x^{\prime}<x$, since otherwise the minimality of $x^{\prime}$ makes it impossible for the trace location exercise for $x^{\prime}$ to have been suspended at stage $e+1$. 
If (ii) holds, we have

$$
A^{e}\left\lceil u^{e}\left(\Phi_{i}^{A}, w\right) \subset\left\langle e A^{s}\right\rangle, \quad \text { but } \not \subset\left\langle e A^{s+1}\right\rangle .\right.
$$

So for some $t$-fix trace $y$ for $x^{\prime}$ say, $t \leq e$, either

$$
y \in\left(A ^ { e } \left\lceilu^{e}\left(\Phi_{i}^{A}, w\right) \cap\left\langle e A^{s+1}\right\rangle-\left\langle e A^{s+1}\right\rangle\right.\right.
$$

or

$$
y \in\left\langle e A^{s+1}\right\rangle \mid u^{e}\left(\Phi_{i}^{A}, w\right)-\left(A^{e} \cup\left\langle e A^{s}\right\rangle\right) .
$$

Again, either $y$ is relocated at stage $s+1$ (which will now include the possibility that the trace location exercise for $x^{\prime}$ was suspended at stage $e+1$, so that we merely extract $y$ from $A^{s+1}$ and do not choose independently of other trace locations a new $x^{\prime}$ trace at stage $\left.s+1\right)$ or $x^{\prime} \in D^{s}-D^{s+1}$. Again, since $x^{\prime} \leq y$, we have $x^{\prime} \leq u^{e}\left(\Phi_{i}^{A}\right)$. And in the former case, we get $x^{\prime}<x$ unless the trace location exercise for $x^{\prime}$ is suspended at stage $e+1$, in which case we have $y \in A^{e}\left\lceil u^{e}\left(\Phi_{i}^{A}, w\right)-\left\langle e A^{s^{\prime}}\right\rangle\right.$ at every stage $s^{\prime} \geq s+1$.

We now obtain from the above that the $x$-trace change at stage $s+1$ originates with either a trace location for some $x^{\prime}<x$ or with some number $v$ being permanently extracted from $A$ where $v \leq u^{e}\left(\Phi_{i}^{A}, w\right)=\rho_{x}^{s}\left(z^{*}\right)$. Also, in the latter case we must have

$$
\rho_{x}^{s}\left(z^{*}\right) \geq x>\rho_{x}^{s+1}\left(z^{*}\right),
$$

giving $x>\rho_{x}^{s^{\prime}}\left(z^{*}\right)$ for all $s^{\prime} \geq s+1$ at which $x \in D^{s^{\prime}}$. But this means the number of $x$-trace relocations is less than or equal to $x+\sum_{x^{\prime}<x}$ (the number of $x^{\prime}$-trace relocations), from which the lemma follows.

Lemma 2. $\Omega, \Gamma$, and $\Lambda$ are consistent p.r. functionals, and

$$
A=\Gamma^{D}, \quad B=\Lambda^{D}, \quad D=\Omega^{A, B},
$$

where $A, B$, are d-r.e. sets.

Proof. By the construction, we define $\Omega^{A, B}(x)=D(x)$ at each stage $s+1>x$, each $x \geq 0$, with $\omega(x)=$ the maximum of the $x$-traces. Since there is an $x$ trace in $A \cup B$ if and only if $x \in D$, at each such stage, $\Omega$ satisfies the lemma.

Again, by the construction, we define $A(y)=\Gamma^{D}(y), B(y)=\Lambda^{D}(y)$ at each stage $s+1>y$ for each $y \geq 0$. Also, it is easily verified that $\gamma(y)$ is bounded with $s+1$. Let $s+1>y$ be the least stage at which we inconsistently define $A(y)=\Gamma^{D}(y)$ (some $y \geq 0$ ). So $y$ must be a trace for some $x$, say, and we have a stage $t+1<s+1$ such that we define

$$
A(y)=\Gamma^{D}(y)=\delta_{t+1}, \quad A(y)=\Gamma^{D}(y)=\delta_{s+1},
$$

say, at stages $t+1, s+1$ where $\delta_{s+1}=1-\delta_{t+1}$ and $D\left\lceil\gamma^{t+1}(y), D\left\lceil\gamma^{s+1}(y)\right.\right.$ are comparable. This means there is some (least) stage $s^{\prime}+1, s+1 \geq s^{\prime}+1>t+1$, at which the trace location for $x$ at stage $s^{\prime}+1$ either results in (a) $y$ being selected as a first $x$-trace $\in A \cup B$, (b) in an $x$-trace change at stage $s^{\prime}+1$, or (c) $x$ being extracted from $D$ at stage $s^{\prime}+1$. We also notice that in (a) or (b) we have

$$
y \in A^{s^{\prime}+1} \Rightarrow y \in A^{t^{\prime}+1}-A^{t+1}
$$

for each $t^{\prime}+1, s+1 \geq t^{\prime}+1 \geq s^{\prime}+1$.

If (a) occurs, $x \in D^{s+1}-D^{t+1}$. $D\left\lceil\gamma^{t+1}(y) \mid D \backslash \gamma^{s+1}(y)\right.$, a contradiction.

But $\gamma^{t+1}(y) \gamma^{s+1}(y) \geq x$, giving 
Similarly, if (c) occurs, $x \in D^{t^{\prime}+1}$ at each stage $t^{\prime}+1<s^{\prime}+1$ at which $y \in A^{t^{\prime}+1}$, and at every stage we have $x \leq \gamma(y)$. Hence $D^{t+1}(x) \neq D^{s+1}(x)$, again contradicting the comparability of $D^{t+1}\left\lceil\gamma^{t+1}(y)\right.$ and $D^{s+1}\left\lceil\gamma^{s+1}(y)\right.$.

On the other hand, (b) implies that the $x$-use string $\rho_{x}^{s^{\prime}+1}$ changes at stage $s+1$. In particular, we have $\rho_{x}^{s^{\prime}+1}(z) \neq \rho_{x}^{s^{\prime}}(z)$, some (least) $z<x$.

Now, arguing as in Lemma 1 , we have that either there is some trace relocation for some $e^{\prime}+1$-fix $x^{\prime}<x$ at stage $s^{\prime}+1$, with $e^{\prime}+1<e+1$, or there is some number $v$ permanently extracted from $A \cup B$ where $v \leq \rho_{x}^{s^{\prime}}(z)$ and $v$ is a trace for some $x^{\prime} \in D^{e+1}, x^{\prime}<x$. Hence we inductively obtain that there is some $x^{\prime \prime}$-trace $v$ permanently extracted from $A \cup B$ at stage $s^{\prime}+1$ where $v \leq \rho_{x^{\prime}}^{s}(z)$, for some $x^{\prime}$, where $x^{\prime} \in D^{e+1}, x^{\prime \prime}<v$, and $x^{\prime \prime}$ has entry stage < that for $x^{\prime}$. We can also assume that the inductive process ends up with this permanent extraction not being due to trace relocation, but due to $x^{\prime \prime}$ being extracted from $D^{s^{\prime}+1}$ at stage $s^{\prime}+1$. But by the definition of $\gamma^{t^{\prime}+1}(y)$, $\gamma^{t^{\prime}+1}(y) \geq \rho_{x^{\prime}}^{t^{\prime}+1}(z)$, for each stage $t^{\prime}+1 \geq e+1$ at which $x^{\prime} \in D^{t^{\prime}+1}$, we again find that the assumed inconsistency in $\Gamma$ is impossible (using the minimality of $\left.s^{\prime}+1\right)$. This completes the proof of Lemma 2 .

We now define injury sets (compare [11, p. 123])

$$
\begin{aligned}
I_{2 i} & =\left\{x \mid \exists s, t, z\left[A^{t}(x) \neq A(x) \& x \leq u^{t}\left(\Phi_{i}^{A}, z\right) \leq \sigma_{2 i}^{s+1}(2 i)\right]\right\}, \\
I_{2 i+1} & =\left\{x \mid \exists s, t, z\left[B^{t}(x) \neq B(x) \& x \leq u^{t}\left(\Phi_{i}^{B}, z\right) \leq \sigma_{2 i+1}^{s+1}(2 i+1)\right]\right\},
\end{aligned}
$$

for each $i \geq 0$. Let

$$
\begin{aligned}
& \mathscr{A}(n) \Leftrightarrow I_{n} \text { is r.e., } \\
& \mathscr{B}(n) \Leftrightarrow R_{n} \text { is satisfied, } \\
& \mathscr{C}(n) \Leftrightarrow \operatorname{Lim}_{x} \operatorname{Lim}_{s} \rho_{x}^{s} \uparrow(n+1) \text { exists }=\rho_{n} \text { (say). }
\end{aligned}
$$

We inductively verify $\mathscr{A}(n), \mathscr{B}(n)$, and $\mathscr{C}(n)$ for all $n$.

Lemma 3. $(\forall m<n) \quad \mathscr{C}(m) \Rightarrow \mathscr{A}(n)$.

Proof. Let $n=2 i$ (the case $n=2 i+1$ is similar).

By $(\forall m<n) \mathscr{C}(m)$ choose a stage $t+1$ such that $\rho_{x}^{s+1}(z) \geq \rho_{n}(z)$ for all $s+1 \geq t+1$, all $x$ with entry stage $e+1<s+1$, all $z<n$. Choose $r$ greater than $n$ and greater than all such $\rho_{n}(z)$. Choose $r^{*} \geq r$ such that $r^{*}$ is greater than every $x$-trace with $x \leq r$. Choose $v+1 \geq t+1$ such that

$$
D \uparrow r=D^{v^{\prime}+1}\left\lceil r \text { and }(A \cup B) \uparrow r^{*}=\left(A^{v^{\prime}+1} \cup B^{v^{\prime}+1}\right) \uparrow r^{*}\right.
$$

for each $v^{\prime}+1 \geq v+1$.

We show that $I_{n} \cap A$ is finite, since $y \in I_{n} \cap A \Rightarrow y \in A^{v+1}$. Let $y \in I_{n} \cap A$. This means we can choose $s, t, z$ such that

$$
y \in\left(A^{s+1} \cap A\right)-A^{t} \& y \leq u^{t}\left(\Phi_{i}^{A}, z\right) \leq \sigma_{n}^{s+1}(n),
$$

and $y$ is an $x$-trace for some $e+1$-fix $x$ with $e+1 \leq s+1$ and $x \in D$. Then at each stage $s^{\prime}+1>s+1$ we do not relocate the trace for $x$ at stage $s^{\prime}+1$. The reason for this can be either

(i) the least $z$ such that $x \leq \rho_{x}^{s^{\prime}+1}(z)$ is the same least $z$ such that $x \leq$ $\rho_{x}^{s^{\prime}}(z)$, or 
(ii) the trace location exercise for $x$ is suspended at stage $s^{\prime}+1$ through some $e^{\prime}+1-\mathrm{fix} x^{\prime}$ (whose trace location is not suspended), $e^{\prime}+1>e+1$, $x^{\prime}<x$, and we do not relocate the trace for $x^{\prime}$ at stage $s^{\prime}+1$.

If (i) holds at each large enough such stage $s^{\prime}+1$, we must have $z<n$ and $x \leq \operatorname{Lim}_{x} \operatorname{Lim}_{s} \rho_{x}^{s}(z)=\rho_{n}(z) \leq r$. But then by choice of $r, r^{*}$, and $v+1$ we have $s=1 \leq v+1$.

If (ii) holds at infinitely many such stages $s^{\prime}+1$, at each such stage there is some such $x^{\prime}$ and hence (since there are only finitely many such $x^{\prime}$ ) such an $x^{\prime} \in D$, so that trace location for $x$ is permanently suspended. Hence in this case (i) now applied to $x^{\prime}$ at all large enough stages. But this means that $x$-trace location was suspended at a stage $\leq v+1$, and in each case it follows that $y \in I_{n} \cap A \Rightarrow y \in A^{v+1}$.

$\mathscr{A}(n)$ immediately follows from the finiteness of $I_{n} \cap A$. This is because if $x \in I_{n}=I_{2 i}$, say, then either $x \in A-A^{t}$, for some $t$, or $x \in A^{t}-A$, for some $t$. So we have $x \in I_{n}$ if and only if either $x \in I_{n} \cap A$ or

$$
\exists s, t, t^{\prime}, z\left[x \in A^{t}-A^{t^{\prime}} \& x \leq u^{t}\left(\Phi_{i}^{A}, z\right) \leq \sigma_{n}^{s+1}(2 i),\right.
$$

and hence $I_{n}$ is r.e. as required.

Lemma 4. $\mathscr{A}(n) \Rightarrow \mathscr{B}(n)$.

Proof. Take $n=2 i$ again. As in Lemma 3, let $v+1$ be such that $y \in I_{n} \cap A \Rightarrow$ $y \in A^{v+1}$ (that is, $R_{n}$ is never injured after stage $v+1$, except by the extraction of traces).

Assume that $D=\Phi_{i}^{A}$, so that $\operatorname{Lim}_{s} l^{s}\left(D, \Phi_{i}^{A}\right)=\infty$. We show that $I_{n} \equiv_{T} D$.

To show $I_{n} \leq_{T} D$, we first use the fact that $A \leq_{T} D$ via $\Gamma$, by Lemma 2 . We can compute $I_{n}$ from $D$ as follows:

To complete $I_{n}(p)$, we first find the largest stage $t^{\prime}$ at which we have

$$
\Gamma^{D}(p) \uparrow \quad \text { or } \Gamma^{D}(p) \downarrow \quad \text { with } D^{t^{\prime}} \uparrow \gamma^{t^{\prime}}(p) \not \subset D .
$$

From this we find all stages $t$ for which $A^{t}(p) \neq A(p)$. For each such stage $t$ we check with the help of $A, \Gamma$, and $D$ whether there is ever a stage $s+1$ such that

$$
\mathscr{U}^{t, s+1}\left(\Phi_{i}^{A}\right)=u^{t}\left(\Phi_{i}^{A}, z\right)
$$

with $p \leq u^{t}\left(\Phi_{i}^{A}, z\right)$, and then whether we ever have

$$
\mathscr{U}^{s+1}\left(\Phi_{i}^{A}\right)=\mathscr{U}^{t, s+1}\left(\Phi_{i}^{A}\right) .
$$

We then decide whether $p \in I_{n}$ using the definitions of $I_{n}$ and $\sigma_{n}^{s+1}(n)$.

To show $D \leq_{T} I_{n}$ we use $D=\Phi_{i}^{A}$ to give an algorithm for computing $D(p)$ from $I_{n}$ :

Look for a stage $t>v+1$ such that

$$
p+1 \leq l^{t}\left(D, \Phi_{i}^{A}\right) \& \sigma_{n}^{t+1}(n) \downarrow \geq u^{t}\left(\Phi_{i}^{A}, p+1\right)
$$

where

$$
\forall w \leq u^{t}\left(\Phi_{i}^{A}, p+1\right)\left[w \in A^{t} \Rightarrow w \notin I_{n}\right] .
$$

Set $D(p)=\Phi_{i}^{A}(p([t]$.

We verify (a) for each $p \geq 0$ such a stage $t$ exists, and (b) given such a $t$, $D(p)=\Phi_{i}^{A}(p)[t]$. 
For (a), let $t^{\prime}$ be a stage $>v+1$ such that

$$
A^{t^{\prime}}\left\lceil u^{t^{\prime}}\left(\Phi_{i}^{A}, p+1\right) \subset A^{s^{\prime}} \text { and } p+1 \leq l^{s^{\prime}}\left(D, \Phi_{i}^{A}\right)\right.
$$

for all $s^{\prime} \geq t^{\prime}$, and take $t=t^{\prime}$. Then

$$
A^{s^{\prime}} \mid u^{t^{\prime}}\left(\Phi_{i}^{A}, p+1\right) \subset\left\langle t A^{s^{\prime}+1}\right\rangle
$$

for all $s^{\prime} \geq t^{\prime}$, which means $u^{t}\left(\Phi_{i}^{A}, p+1\right)$ is valid at each stage $s^{\prime}+1 \geq t+1$. Hence

$$
\sigma_{n}^{s^{\prime}+1}(n) \downarrow \geq \mathscr{U}^{s^{\prime}+1}\left(\Phi_{i}^{A}\right) \geq \mathscr{U}^{t, s^{\prime}+1}\left(\Phi_{i}^{A}\right) \geq u^{t}\left(\Phi_{i}^{A}, p+1\right)
$$

for each $s^{\prime}+1 \geq t+1$. Also, by the choice of $t$ we have for each $w \leq$ $u^{t}\left(\Phi_{i}^{a}, p+1\right)$ that if $w \in A^{t}$ then $w \in A$, and hence (since $\left.\left.t+1>v+1\right)\right) w \notin$ $I_{n}$. So (a) is proved.

For (b), we inductively verify that for infinitely many stages $s^{\prime}+1>t$

$$
p+1 \leq l^{s^{\prime}}\left(D, \Phi_{i}^{A}\right) \& \sigma_{n}^{s^{\prime}+1}(n) \downarrow \geq u^{t}\left(\Phi_{i}^{A}, p+1\right)=u^{s^{\prime}}\left(\Phi_{i}^{A}, p+1\right) .
$$

$D(p)=\Phi_{i}^{A}(p)[t]$ will then follow from $D=\Phi_{i}^{A}$.

In order to get a contradiction, assume that there is a largest such stage $s^{\prime}+1=s^{*}+1$, say. This means that at every stage $s^{\prime}+1>s^{*}+1$ we have

$$
u^{t}\left(\Phi_{i}^{A}, z\right) \neq u^{s^{\prime}}\left(\Phi_{i}^{A}, z\right)
$$

for some $z \leq p+1$, and without loss of generality, we can assume in this case that at every stage $s^{\prime}+1>s^{*}+1$ we have

$$
u^{t}\left(\Phi_{i}^{A}, p+1\right) \neq u^{s^{\prime}}\left(\Phi_{i}^{A}, p+1\right) .
$$

This means that at each such stage $s^{\prime}+1$ there is some $x$-trace $y \leq$ $\left.u^{t}\left(\Phi_{i}^{A}\right), p+1\right)$, for some $x \geq 0$, for which $A^{t}(y) \neq A^{s^{\prime}}(y)$. So there is some such $y \leq u^{t}\left(\Phi_{i}^{A}, p+1\right)$ for which $A^{t}(y) \neq A(y)$. But by the definition of $I_{n}$ this means $y \in I_{n}$. And by the choice of $v+1$ and $t$ we have $y \leq u^{t}\left(\Phi_{i}^{A}, p+1\right) \Rightarrow y \neq I_{n}$, which is the required contradiction.

Lemma 5. $[((\forall m<n) \mathscr{C}(m)) \& \mathscr{B}(n)] \Rightarrow \mathscr{C}(n)$.

Proof. Assume $R_{n}$ is satisfied, $n=2 i$ again, and that $\operatorname{Lim}_{x} \operatorname{Lim}_{\mathrm{s}} \rho_{x}^{s}(z)$ exists for each $z<n$. We need to show that $\operatorname{Lim}_{x} \operatorname{Lim}_{s} \rho_{x}^{s}(n)$ exists.

If $D \neq \Phi_{i}^{A}$ then $\liminf _{s} u^{s}\left(\Phi_{i}^{A}\right)$ exists $=u\left(\Phi_{i}^{A}\right)$, say. We show that

$$
\operatorname{Lim}_{x} \operatorname{Lim}_{s} \rho_{x}^{s}(n)=u\left(\Phi_{i}^{A}\right) .
$$

By definition, if $s>e$,

$$
\begin{aligned}
\rho_{x}^{s}(n) & =\mathscr{U}^{e, s+1}\left(\Phi_{i}^{A}\right) \\
& =\max \left\{u^{e}\left(\Phi_{i}^{A}, z\right) \mid z \leq l^{e}\left(D, \Phi_{i}^{A}\right) \& u^{e}\left(\Phi_{i}^{A}, z\right)\right.
\end{aligned}
$$

is valid at stage $s+1\}$.

Let $v+1$ be as in Lemma 3. Then if $e>v+1$, and if $y \leq u^{e}\left(\Phi_{i}^{A}, z\right)$ and $u^{e}\left(\Phi_{i}^{A} z\right)$ is valid at a stage $s+1>e$ but $y \in\left\langle e A^{s+1}\right\rangle-A^{e}$, then $y \notin A$. Hence if $z \leq l^{e}\left(D, \Phi_{i}^{A}\right)$ and $u^{e}\left(\Phi_{i}^{A}, z\right)$ is valid at infinitely large stages $s+1$ we have $A^{e} \uparrow u^{e}\left(\Phi_{i}^{A}, z\right) \subset A$. It follows that 


$$
\operatorname{Lim}_{s} \rho_{x}^{s}(n)=\max \left\{u^{e}\left(\Phi_{i}^{A}, z\right)\left|z \leq l^{e}\left(D, \Phi_{i}^{A}\right) \& A^{e}\right| u^{e}\left(\Phi_{i}^{A}, z\right) \subset A\right\}
$$

from which Lemma 5 follows.

We now get $\mathscr{A}(n), \mathscr{B}(n)$, and $\mathscr{C}(n)$ inductively for all $n$.

Now say we have the theorem for some $n \geq 2$, and $\mathbf{d}$ is an $(n+1)$-r.e. degree. Then either $\mathbf{d}$ is n-r.e., in which case it can be split, or $\mathbf{d}$ is properly $(n+1)$-r.e. (that is, $(n+1)$-r.e. but not n-r.e.). In this case we can carry out the above construction but with $D((n+1)$-r.e., deriving injury sets that are n-.r.e instead of just r.e. and adjusting the use of the traces to $n$ instead of 1 . This completes the proof of the theorem.

As an application of the above, one might follow Arslanov's idea [1] of using splitting to prove density of certain subclasses of the n-r.e. degrees (Sacks' original approach to proving the density of the r.e. degrees, it seems), perhaps extending the techniques of [10] where it is shown that relative splitting is always possible within the low 2 degrees.

Completely untouched by any existing result remains

Downey's Conjecture [5]. The theories of the n-r.e. degrees for each $n \geq 2$ are elementary equivalent.

The above theorem, like previous work, presents a situation in which the r.e. case is the special one (the injury set is finite), and the n-r.e. case, $n>2$, follows from the d-r.e. one.

\section{REFERENCES}

1. M. M. Arslanov, Structural properties of the degrees below $\mathbf{0}^{\prime}$, Dokl. Akad. Nauk. SSSR 283 (1985), 270-273.

2. N. R. Bukaraev, On T-degrees of differences of recursively enumerable sets, Izv. Vyssh. Uchebn. Zaved. Mat. 228 (1981), 40-49 (Russian); English transl.: Sov. Math. (IzVUZ) 25 (1981), 40-52.

3. S. B. Cooper, L. Harrington, A. H. Lachlan, S. Lempp, and R. I. Soare, The d-r.e. degrees are not dense, to appear in Annals of Pure and Applied Logic.

4. S. B. Cooper, S. Lempp, and P. Watson, Weak density and cupping in the d-r.e. degrees, Israel J. Math. 67 (1989), 137-152.

5. R. G. Downey, D-r.e. degrees and the nondiamond theorem, Bull. London Math. Soc. 21 (1989), 43-50.

6. Sh. T. Ishmuchametov, On differences of recursively enumerable sets, Izv. Vyssh. Uchebn. Zaved. Mat. 279 (1985), 3-12. (Russian)

7. C. G. Jockusch, Jr. and R. A. Shore, Pseudo jump operators I: The R. E. case, Trans. Amer. Math. Soc. 275 (1983), 599-609.

8. P. Odifreddi, Classical recursion theory, North-Holland, Amsterdam, New York, Tokyo and Oxford, 1989.

9. G. E. Sacks, On the degrees less than $0^{\prime}$, Ann. of Math. (2) 77 (1963), 211-231.

10. R. A. Shore and T. A. Slaman, Working below a low 2 recursively enumerable degree (to appear).

11. R. I. Soare, Recursively enumerable sets and degrees, Springer-Verlag, Berlin, New York, and London, 1987. 